\title{
Sub specie aeternitatis
}

\author{
Sub specie aeternitatis
}

\section{Frank Thomas Sautter}

Resumo: A formalização de noções pré-teóricas não é uma ciência, mas uma arte. Isso se evidencia quando temos de decidir o que é indispensável e o que é prescindível na passagem do intuitivo para o formal. Exemplifico esse tipo de dificuldade com a formalização de uma noção intuitiva de coleção finita de objetos. A observância de maior fidelidade à noção préteórica, mesmo que desnecessária da perspectiva formal, resultou em duas novas definições de conjunto finito.

Palavras-chave: Definição. Finito. Formal. Pré-teórico. Teoria dos Conjuntos.

\begin{abstract}
The formalization of pre-theoretical notions is not a science, but an art. This becomes evident when we have to decide what is indispensable and what is essential in the transition from the intuitive to the formal. I exemplify this type of difficulty with the formalization of an intuitive notion of a finite collection of objects. The observance of greater fidelity to the pretheoretical notion, even if unnecessary from a formal perspective, resulted in two new definitions of finite set.
\end{abstract}

Keywords: Definition. Finite. Formal. Pre-theoretical. Set theory.

Data de recebimento: $19 / 04 / 2020$

Data de aceite: $07 / 10 / 2020$

DOI: $10.23925 / 2316-5278.2020 v 21 \mathrm{i} 2 \mathrm{p} 300-306$

"Um dia você aprende que não pode ter tudo o que quer..."

Mário Quintana

\section{Introdução}

Hansson (2000, p. 164) esclarece que o processo de formalização de noções préteóricas demanda duas etapas de idealização. Na primeira etapa nos movemos da linguagem comum, na qual a noção pré-teórica é expressa, para uma linguagem regimentada, na qual são fixadas, por exemplo, a categoria das variáveis utilizadas,

* Universidade Federal de Santa Maria - Santa Maria, RS, Brasil. Professor titular de lógica na UFSM. E-mail: ftsautter@ufsm.br. 
o escopo dessas variáveis etc. Na segunda etapa nos movemos de uma linguagem regimentada para a uma linguagem lógica ou matemática. Hansson (2006, p. 20) sugere que esse processo de formalização requer um método de sucessivos melhoramentos, no qual a estreiteza ou a amplitude da noção formalizada em relação à noção pré-teórica é paulatinamente corrigida. Neste breve trabalho forneço um exemplo adicional da difícil arte de formalizar: o processo de formalização de uma noção pré-teórica de coleção finita de objetos. O exemplo abrevia uma etapa, desde que nos movemos diretamente da linguagem comum para a linguagem conjuntista, mas há diversos movimentos adicionais em direção a uma formalização mais fiel à noção pré-teórica dada. O exemplo sugere que é possível haver múltiplas implementações da mesma noção pré-teórica que não diferem quanto ao essencial, e que uma maior ou menor fidelidade à noção pré-teórica é consoante ao gosto do freguês.

\section{A noção pré-teórica e sua formalização inicial}

Winston Alarcón Athens (apud SAUTTER, 1995, p. 45ss.) propôs uma caracterização de coleção finita de objetos que captura a seguinte intuição: uma coleção de objetos é finita, se ela se esgota ao retirarem-se seus elementos um a um. ${ }^{1}$ Essa caracterização é formalizada por Alarcón Athens, no âmbito da Teoria dos Conjuntos, mediante o seguinte par de definições:

Definição 1. A família $F$ de subconjuntos de C é unitariamente decrescente se, e somente se, para todo conjunto não-vazio $G \in F$ existe um conjunto $H \in F$ tal que $\mathrm{H}=\mathrm{G}-\{\mathrm{g}\}$ para algum $\mathrm{g} \in \mathrm{G}^{2}$

Definição 2. Um conjunto $\mathrm{C}$ é finito ${ }_{1}$ se, e somente se, $\varnothing \in \mathrm{F}$ para toda família nãovazia $\mathrm{F}$ de subconjuntos de $\mathrm{C}$ unitariamente decrescente.

Sautter (1995, p. 45) utiliza a seguinte construção para demonstrar que o conjunto dos números naturais $\mathrm{N}$ não é finito ${ }_{1}$ :

a) Para todo $\mathrm{n} \in \mathrm{N}, \mathrm{C}_{\mathrm{n}}=\{\mathrm{m} \in \mathrm{N}: \mathrm{m} \geq \mathrm{n}\}$

b) $F=\left\{C_{n}: n \in N\right\}$, ou seja, $F$ é a coleção de segmentos finais da série dos números naturais em sua ordem habitual.

F é uma família não-vazia de subconjuntos de $\mathrm{N}$ unitariamente decrescente e é tal que $\varnothing \mathrm{F}$.

1 O mesmo não ocorre com uma coleção infinita de objetos? A resposta é negativa. Considere, por exemplo, o Hotel de Hilbert, uma edificação com infinitos pavimentos (EWALD; SIEG, 2013, p. 730): se retiramos o rés-do-chão, ainda há uma infinidade de pavimentos na nova edificação; se retiramos o rés-do-chão da nova edificação, ainda há uma infinidade de pavimentos; e assim por diante.

2 A definição original de Alarcón Athens não menciona o conjunto $C$. De fato, a menção de $C$ é prescindível no definiendum, porque o definiens não o utiliza. Entretanto, essa definição é somente auxiliar à próxima definição e, naquela, $C$ é utilizado no definiendum, o que justifica a decisão aqui adotada. 
Uma caracterização intuitiva alternativa de coleção finita de objetos recorre ao acréscimo de objetos ao invés de decréscimo: uma coleção de objetos é finita, se ela é obtida ao apor um a um seus elementos. ${ }^{3}$ Esta caracterização alternativa é formalizada por Alarcón Athens, no âmbito da Teoria dos Conjuntos, mediante o seguinte par de definições:

Definição 3. A família $\mathrm{F}$ de subconjuntos de C é unitariamente crescente se, e somente se, para todo conjunto $\mathrm{G} \in \mathrm{F}$, distinto de $\mathrm{C}$, existe um conjunto $\mathrm{H} \in \mathrm{F}$ tal que $\mathrm{H}=\mathrm{G} \cup\{\mathrm{g}\}$ para algum $\mathrm{g} \in \mathrm{C}-\mathrm{G} .^{4}$

Definição 4. Um conjunto $\mathrm{C}$ é finito ${ }_{2}$ se, e somente se, $\mathrm{C} \in \mathrm{F}$ para toda família nãovazia $\mathrm{F}$ de subconjuntos de $\mathrm{C}$ unitariamente crescente.

Sautter (1995, p. 46) utiliza a seguinte construção para demonstrar que o conjunto dos números naturais $\mathrm{N}$ não é finito ${ }_{2}$ :

a) Para todo $n \in N, C_{n}=\{m \in N: m<n\}$

b) $\mathrm{F}=\left\{\mathrm{C}_{\mathrm{n}}: \mathrm{n} \in \mathrm{N}\right\}$, ou seja, $\mathrm{F}$ é a coleção de segmentos iniciais da série dos números naturais em sua ordem habitual.

F é uma família não-vazia de subconjuntos de $\mathrm{N}$ unitariamente crescente e é tal que $\mathrm{N} \notin \mathrm{F}$.

Sautter (1995, p. 47-48) demonstra a equivalência das noções de finito (Definição 2) e de finito $_{2}$ (Definição 4). Sautter (1995, p. 48-49) também demonstra a equivalência dessas duas noções de finito com a noção aritmética usual de finito, ${ }^{5}$ prescindindo da utilização do Axioma de Escolha e, mesmo, de formas fracas do Axioma da Escolha. ${ }^{6}$ Isso basta do ponto de vista formal. Entretanto, essas definições ainda não fazem justiça à caracterização intuitiva. Um exemplo ajudará a ilustrar a situação.

Seja o conjunto $\mathrm{C}=\{\mathrm{a}, \mathrm{b}, \mathrm{c}\}$ e a família $\mathrm{F}=\{\mathrm{C},\{\mathrm{a}, \mathrm{b}\},\{\mathrm{b}, \mathrm{c}\},\{\mathrm{a}\},\{\mathrm{b}\}, \varnothing\}$ de subconjuntos de C. F é unitariamente decrescente (Definição 1) e também é unitariamente crescente (Definição 3). Consideremos a caracterização intuitiva de retirada, um a um, dos objetos de C. Começando com $\mathrm{C}$ em F, retiramos um objeto e obtemos... Aqui está o problema: retiramos c e obtemos $\{a, b\}$, mas se retiramos

3 O Hotel de Hilbert, por exemplo, não poderia ser edificado desse modo - edificar o résdo-chão, apor um pavimento, apor outro pavimento, e assim por diante -, pois sempre teríamos apenas uma quantidade finita de pavimentos.

4 Aqui, ao contrário da definição de família de conjuntos unitariamente decrescente, a menção de $C$ é imprescindível no definiendum, porque o definiens o utiliza.

5 Um conjunto é finito, no sentido aritmético usual, se, e somente se, existe uma correspondência biunívoca entre o conjunto e um segmento inicial da série dos números naturais, em sua ordem habitual (ver SAUTTER, 1995, p. 4).

6 Em rigor, Sautter (1995, p. 48-49) utiliza as noções de conjunto finito de Tarski e de Russell, cuja equivalência com a noção aritmética usual, prescindindo do Axioma da Escolha e, mesmo, de formas fracas do Axioma da Escolha, é bem conhecida na literatura. Que o Axioma da Escolha ou formas fracas dele sejam prescindiveis é um importante esclarecimento, porque não há um consenso entre os especialistas sobre a validade universal ou não do Axioma da Escolha e de formas fracas do Axioma da Escolha. 
a obtemos $\{b, c\}$. Uma família de subconjuntos unitariamente decrescente admite múltiplas retiradas unitárias de um mesmo conjunto, mas isso é contrário ao espírito da caracterização intuitiva. ${ }^{7}$

\section{Aperfeiçoando a formalização inicial}

Uma aproximação melhor da caracterização intuitiva é dada pelo seguinte par de definições de conjunto finito:

Definição 5. A família $\mathrm{F}$ de subconjuntos de $\mathrm{C}$ é minimamente unitariamente decrescente se, e somente se, para todo conjunto não-vazio $G \in F$ existe um único conjunto $\mathrm{H} \in \mathrm{F}$ tal que $\mathrm{H}=\mathrm{G}-\{\mathrm{g}\}$ para $\mathrm{g} \in \mathrm{G}$.

Definição 6. Um conjunto é finito ${ }_{3}$ se, e somente se, $\varnothing \in \mathrm{F}$ para toda família nãovazia $\mathrm{F}$ de subconjuntos de $\mathrm{C}$ minimamente unitariamente decrescente. ${ }^{8}$

Definição 7. A família $\mathrm{F}$ de subconjuntos de $\mathrm{C}$ é minimamente unitariamente crescente se, e somente se, para todo conjunto $\mathrm{G} \in \mathrm{F}$, distinto de $\mathrm{C}$, existe um único conjunto $\mathrm{H} \in \mathrm{F}$ tal que $\mathrm{H}=\mathrm{G} \cup\{\mathrm{g}\}$ para $\mathrm{g} \in \mathrm{C}-\mathrm{G}$.

Definição 8. Um conjunto é finito ${ }_{4}$ se, e somente se, $\mathrm{C} \in \mathrm{F}$ para toda família nãovazia $\mathrm{F}$ de subconjuntos de $\mathrm{C}$ minimamente unitariamente crescente.?

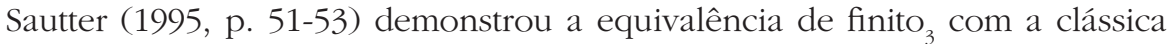
definição de Dedekind, segundo a qual um conjunto é finito se, e somente se, não há correspondência biunívoca entre ele e qualquer de seus subconjuntos próprios. Essa demonstração prescinde do Axioma da Escolha e de formas fracas do Axioma da Escolha. ${ }^{10}$

As noções de finito $_{3}$ e finito $_{4}$ corrigem uma infidelidade à noção intuitiva, acima apontada, das noções de finito e finito $_{2}$, respectivamente, mas ainda não fazem justiça à caracterização intuitiva de coleção finita de objetos, dada no início do trabalho. Um novo exemplo ajudará a esclarecer qual é o problema remanescente.

Seja novamente $\mathrm{C}=\{\mathrm{a}, \mathrm{b}, \mathrm{c}\}$ e, dessa vez, a família $\mathrm{F}=\{\{\mathrm{b}, \mathrm{c}\},\{\mathrm{b}\}, \varnothing\}$ de subconjuntos de C. F é minimamente unitariamente decrescente (Definição 5). Consideremos a caracterização intuitiva de retirada, um a um, dos objetos de C. C não está em F e, portanto, sequer podemos iniciar o processo de retirada, um a um, de elementos de C, o que é contrário ao espírito da caracterização intuitiva. ${ }^{11}$

7 A mesma objeção pode ser levantada em relação à noção de família de subconjuntos unitariamente crescente.

8 Esta definição de conjunto finito é uma contribuição original de Sautter (1995).

9 Esta definição de conjunto finito também é uma contribuição original de Sautter (1995).

10 A adaptação da demonstração à prova da equivalência de finito ${ }_{4}$ e finito no sentido de Dedekind não apresenta maiores dificuldades.

11 A mesma objeção pode ser levantada em relação à noção de família de subconjuntos minimamente unitariamente crescente. Por exemplo, $F=\{\{b\},\{b, c\}, C\}$ é uma família de 


\section{Melhorando 0 aperfeiçoamento}

Famílias de subconjuntos minimamente unitariamente decrescentes são casos particulares de famílias de subconjuntos unitariamente decrescentes, e famílias de subconjuntos minimamente unitariamente crescentes são casos particulares de famílias de subconjuntos unitariamente crescentes. O seguinte par de definições utiliza famílias de subconjuntos ainda mais restritas (e tão próximas quanto possível da noção pré-teórica), a saber, famílias de subconjuntos unitária, mínima e completamente decrescentes são casos particulares de famílias de subconjuntos minimamente unitariamente decrescentes, e famílias de subconjuntos unitária, mínima e completamente crescentes são casos particulares de famílias de subconjuntos minimamente unitariamente crescentes:

Definição 9. A família $\mathrm{F}$ de subconjuntos de C é unitária, mínima e completamente decrescente se, e somente se, $\mathrm{C} \in \mathrm{F}$ e para todo conjunto não-vazio $\mathrm{G} \in \mathrm{F}$ existe um único conjunto $H \in F$ tal que $H=G-\{g\}$ para $g \in G$.

Definição 10. Um conjunto $C$ é finito ${ }_{5}$ se, e somente se, $\varnothing \in F$ para toda família $F$ de subconjuntos de C unitária, mínima e completamente decrescente. ${ }^{12}$

Definição 11. A família F de subconjuntos de C é unitária, mínima e completamente crescente se, e somente se, $\varnothing \in \mathrm{F}$ e para todo conjunto $\mathrm{G} \in \mathrm{F}$, distinto de $\mathrm{C}$, existe um único conjunto $\mathrm{H} \in \mathrm{F}$ tal que $\mathrm{H}=\mathrm{G} \cup\{\mathrm{g}\}$ para $\mathrm{g} \in \mathrm{C}-\mathrm{G}$.

Definição 12. Um conjunto $C$ é finito $_{6}$ se, e somente se, $C \in F$ para toda família $F$ de subconjuntos de C unitária, mínima e completamente crescente. ${ }^{13}$

Nada de essencial, do ponto de vista formal, ganha-se ou perde-se na comparação entre finito $_{1}$, finito $_{3}$ e finito ${ }_{5}$, e entre finito $_{2}$, finito e finito $_{6}$. Demonstrarei, a título de ilustração, a equivalência das definições de finito ${ }_{3}$, que utiliza famílias de subconjuntos minimamente unitariamente decrescentes, e de finito ${ }_{5}$, que utiliza famílias de subconjuntos unitária, mínima e completamente decrescentes:

Trivialmente, todo conjunto finito também é finito $_{5}$, porque as famílias de subconjuntos unitária, mínima e completamente decrescentes são casos particulares de famílias de subconjuntos minimamente unitariamente decrescentes.

subconjuntos de $C=\{a, b, c\}$ minimamente unitariamente crescente, mas o processo de acréscimo de unidades não se inicia no espírito da caracterização intuitiva, ou seja, ele não se inicia com o conjunto vazio.

12 Ao contrário das definições anteriores, aqui não é exigido que a família seja não-vazia, porque isso já está imposto na definição de família unitária, mínima e completamente decrescente ao exigirmos que $C \in F$. Essa definição de conjunto finito é uma contribuição original deste trabalho.

13 Aqui também não é exigido que a família seja não-vazia, porque isso já está imposto na definição de família unitária, mínima e completamente crescente ao exigirmos que $\varnothing \in$ $F$. Esta definição de conjunto finito também é uma contribuição original deste trabalho. 
Fornecerei o esboço da demonstração, por contraposição, que todo conjunto finito também é finito $_{3}$. Seja $\mathrm{C}$ um conjunto que não é finito ${ }_{3}$, ou seja, há uma família não-vazia $\mathrm{F}$ de subconjuntos de $\mathrm{C}$ minimamente unitariamente decrescente tal que $\varnothing \notin \mathrm{F}$. Seja $\mathrm{A}_{\mathrm{F}}=\cup \mathrm{F}$, ou seja, $\mathrm{A}_{\mathrm{F}}$ é o conjunto dos elementos de $\mathrm{C}$ que ocorrem em algum subconjunto de $\mathrm{F}$. F é uma família de subconjuntos de $\mathrm{A}_{\mathrm{F}}$ unitária, mínima e completamente decrescente. Portanto, $\mathrm{A}_{\mathrm{F}}$ não é finito ${ }_{5} . \mathrm{A}_{\mathrm{F}} \subset \mathrm{C}$, o que implica que C não é finito.

\section{Considerações finais}

Não é difícil demonstrar os seguintes resultados:

Teorema 1. Se C é um conjunto finito ${ }_{3}$, então toda família de subconjuntos de C unitária, mínima e completamente crescente é unitária, mínima e completamente decrescente.

Teorema 2. Se C é um conjunto finito, , então toda família de subconjuntos de C unitária, mínima e completamente decrescente é unitária, mínima e completamente crescente.

A lição que esses dois teoremas nos oferecem é que, embora tenhamos sido bem sucedidos em representar mudança (acréscimo ou decréscimo de elementos) mediante o imutável (conjuntos), a representação foi capaz de capturar o tempo sem ser capaz de capturar a seta do tempo, ou seja, dada simplesmente uma família de subconjuntos de C unitária, mínima e completa, não somos capazes de determinar se a intenção é descrever um acréscimo de elementos, a começar do conjunto vazio, ou um decréscimo de elementos, a começar de C. Essas duas últimas formalizações têm os seus limites, elas somente são capazes de nos oferecer uma visão, por assim dizer, sub specie aeternitatis daquilo que se passa. As primeiras definições, a despeito da não integralidade na captura da noção pré-teórica, tinham ao menos essa qualidade de diferenciar acréscimo de decréscimo, ao menos em alguns casos. Por exemplo, $\mathrm{F}=\{\{\mathrm{a}, \mathrm{b}, \mathrm{c}\},\{\mathrm{a}, \mathrm{b}\}\}$ é uma família de subconjuntos de $\{\mathrm{a}$, b, c\} unitariamente crescente, mas não é unitariamente decrescente! A situação toda exemplifica uma cama de Procusto, ou, como disse o poeta: "Um dia você aprende que não pode ter tudo o que quer...".

\section{Referências}

EWALD, William; SIEG, Wilfried (Eds.). David Hilbert's lectures on the foundations of Arithmetic and Logic 1917-1933. Berlin: Springer, 2013.

HANSSON, Sven Ove. Formalization in Philosophy. The Bulletin of Symbolic Logic, Cambridge, v. 6, n. 2, p. 162-175, Jun. 2000. DOI: https://doi.org/10.2307/421204.

HANSSON, Sven Ove. How to define: a tutorial. Princípios: revista de filosofia, Natal, v. 13, n. 19-20, p. 5-30, 2006. Disponível em: <https://periodicos.ufrn.br/principios/ article/view/508/440.> Acesso em: 18 Abr. 2020. 
SAUTTER, Frank Thomas. Definições de conjunto finito. Dissertação (Mestrado em Filosofia) - Universidade Estadual de Campinas, Instituto de Filosofia e Ciências Humanas, Campinas, SP. 1995. Disponível em: <http://www.repositorio.unicamp. br/handle/REPOSIP/278861>. Acesso em: 18 Abr. 2020. 